\title{
3D Urban Remote Sensing using Dual-baseline POL-InSAR Images at L-Band
}

\author{
Stefan Sauer*, Laurent Ferro-Famil ${ }^{\dagger}$, Andreas Reigber* and Eric Pottier ${ }^{\dagger}$ \\ * German Aerospace Center (DLR), Microwaves and Radar Institute, \\ 82234 Oberpfaffenhofen-Wessling, Germany \\ Tel./Fax: +49 8153 28-2397/1449, email: stefan.sauer@dlr.de \\ $\dagger$ University of Rennes 1, IETR Laboratory, SAPHIR Team, \\ Bat. 11D, 263 Avenue General Leclerc, CS 74205, 35042 Rennes Cedex, France
}

\begin{abstract}
This paper generalizes a multibaseline interferometric SAR signal model to the polarimetric configuration. Based on this formulation, two high-performance array signal processing techniques are adapted to analyze multibaseline POL-InSAR observations. These new methods enhance the height estimation of scatterers by calculating optimal polarization combinations and allow the determination of their physical characteristics. Applying the algorithms to urban environments, the building layover problem is resolved by means of polarimetric dualbaseline InSAR measurements: Up to two components within one azimuth-range resolution cell are separated. The techniques are tested using dual-baseline Pol-InSAR data acquired by DLR's E-SAR system over Dresden city.
\end{abstract}

\section{INTRODUCTION}

Interferometric SAR (InSAR) is a technique to determine the height location of reflectors, whereas their physical properties can be extracted by SAR polarimetry. A first approach to estimate the vertical location of scattering mechanisms using single-baseline POL-InSAR data has been introduced in [1]. Recently, polarimetric spectral analysis methods have been applied to multibaseline (MB) Pol-InSAR data of urban areas [2], [3], [4]. This paper presents a new way of analyzing polarimetric multibaseline InSAR observations by adapting two array signal processing techniques to this scenario. In section II, the conventional single polarization signal model, the MUSIC algorithm and an ML estimator for MB InSAR height estimation are outlined. Section III describes the generalization to the fully polarimetric MB InSAR set-up: The signal model is adapted to deal with four polarization channels and subsequently the MUSIC and ML estimators are formulated in a rigorous mathematical way and their features are described. Finally, experimental results are shown in section IV: The methods are applied to solve the building layover problem using fully polarimetric MB InSAR data of Dresden city acquired by DLR's E-SAR system.

\section{Multibaseline InSAR Spectral Analysis ALGORITHMS}

This section describes a single polarization multibaseline InSAR signal model and two MB InSAR spectral analysis techniques: the MUSIC algorithm and an ML estimator.

\section{A. Multibaseline InSAR Signal Model}

The single polarization multibaseline InSAR received signal with $p$ sensors can be modeled as

$$
\begin{aligned}
\mathbf{y}(n) & =\mathbf{t}+\mathbf{c}(n)+\mathbf{v}(n) \\
& =\sum_{i=1}^{N_{s_{t}}} \sqrt{\tau_{t_{i}}} \exp \left(j \psi_{i}\right) \mathbf{a}\left(z_{t_{i}}\right) \\
& +\sum_{j=1}^{N_{s_{c}}} \sqrt{\tau_{c_{j}}} \mathbf{x}_{j}(n) \odot \mathbf{a}\left(z_{c_{j}}\right)+\mathbf{v}(n)
\end{aligned}
$$

with $n=1, \ldots, N$, the number of looks $N$, and the SchurHadamard product $\odot$ (elementwise multiplication). The MB InSAR received data vector $\mathbf{y}(n) \in \mathbb{C}^{p}$ is assumed to be a Gaussian random process with nonzero mean and covariance matrix $\mathbf{R} \in \mathbb{C}^{p \times p}$, i.e., $\mathbf{y}(n) \sim \mathcal{N}_{\mathbb{C}}(\boldsymbol{\mu}, \mathbf{R})$. The first term, $\mathbf{t}$, is highly coherent and can be associated to a deterministic or almost deterministic target [5]. The second contribution, $\mathbf{c}(n)$, represents the response of distributed environments including the SAR speckle effect as multiplicative noise [6]. The additive white Gaussian noise $\mathbf{v}(n) \in \mathbb{C}^{p}$ has zero mean and power $\sigma_{v}^{2}$, i.e., $\mathbf{v}(n) \sim \mathcal{N}_{\mathbb{C}}\left(\mathbf{0}, \sigma_{v}^{2} \mathbf{I}\right)$. The number of backscattering sources $N_{s_{t}}$ and $N_{s_{c}}$ of the coherent and incoherent component, respectively, are assumed to be known. The total number of scatterers is $N_{s}=N_{s_{t}}+N_{s_{c}}$. The reflectivity $\tau$ and the height $z$ of the scatterers as well as the complex argument $\psi$ are considered to be deterministic unknown quantities. The steering vector $\mathbf{a}(z) \in \mathbb{C}^{p}$ for a general acquisition geometry is represented as

$$
\mathbf{a}(z)=\left[1, \exp \left(j \kappa_{z_{2}} z\right), \ldots, \exp \left(j \kappa_{z_{p}} z\right)\right]^{T}
$$

with the vertical wavenumber $\kappa_{z_{i}}=\frac{4 \pi}{\lambda} \frac{B_{\perp}^{(i)}}{r^{(i)} \sin \theta^{(i)}}$. The multiplicative noise $\mathbf{x}_{i}(n) \in \mathbb{C}^{p}$ is a Gaussian random vector with zero mean and covariance matrix $\mathbf{C}_{i}=E\left\{\mathbf{x}_{i}(n) \mathbf{x}_{i}^{H}(n)\right\}$ where $H$ denotes transpose, complex conjugate. This model does not take multipath effects into account. The sample covariance matrix $\hat{\mathbf{R}} \in \mathbb{C}^{p \times p}$ is computed by

$$
\hat{\mathbf{R}}=\frac{1}{N} \sum_{n=1}^{N} \mathbf{y}(n) \mathbf{y}^{H}(n) .
$$




\section{B. Classical MUSIC Algorithm}

Let $N_{s}$ denote the supposed total number of scatterers. Then the number of eigenvalues of the noise subspace equals $q=p-N_{s}$, and the matrix $\mathbf{E}_{q} \in \mathbb{C}^{p \times q}$ of the corresponding eigenvectors spans this subspace. The pseudospectrum of the single polarization MUSIC method [7] can be calculated by

$$
\hat{P}_{M U}^{S P}(z)=\frac{1}{\mathbf{a}^{H}(z) \mathbf{E}_{q} \mathbf{E}_{q}^{H} \mathbf{a}(z)} .
$$

The pseudospectrum peak locations indicate the height of the scatterers. The subspace spanned by the noise eigenvectors must be at least of dimension one $\left(p \geq N_{s}+1\right)$.

\section{ML Estimator}

Wax [8] proposed an ML estimator based on the stochastic signals model which, unlike MUSIC [9], remains optimal even for correlated signals. Let $\mathbf{A}(\mathbf{z}) \in \mathbb{C}^{p \times N_{s}}$ be the steering vector matrix of the $N_{s}$ sources

$$
\mathbf{A}(\mathbf{z})=\left[\mathbf{a}\left(z_{1}\right), \ldots, \mathbf{a}\left(z_{N_{s}}\right)\right]
$$

with the height vector $\mathbf{z}=\left[z_{1}, \ldots, z_{N_{s}}\right]^{T} \in \mathbb{R}^{N_{s}}$. The projection matrix $\mathbf{P}_{\mathbf{A}(\mathbf{z})} \in \mathbb{C}^{p \times p}$ onto the signal subspace is defined by

$$
\mathbf{P}_{\mathbf{A}(\mathbf{z})}=\mathbf{A}(\mathbf{z})\left(\mathbf{A}^{H}(\mathbf{z}) \mathbf{A}(\mathbf{z})\right)^{-1} \mathbf{A}^{H}(\mathbf{z})
$$

and the orthogonal projection $\mathbf{P}_{\mathbf{A}(\mathbf{z})}^{\perp} \in \mathbb{C}^{p \times p}$ onto the noise subspace is

$$
\mathbf{P}_{\mathbf{A}(\mathbf{z})}^{\perp}=\mathbf{I}-\mathbf{P}_{\mathbf{A}(\mathbf{z})} .
$$

Using the eigenvalues $l_{1}^{S} \geq \ldots \geq l_{N_{s}}^{S}$ of $\mathbf{P}_{\mathbf{A}(\mathbf{z})} \hat{\mathbf{R}} \mathbf{P}_{\mathbf{A}(\mathbf{z})}$ and the eigenvalues $l_{1}^{N} \geq \ldots \geq l_{p-N_{s}}^{N}$ of $\mathbf{P}_{\mathbf{A}(\mathbf{z})}^{\perp} \hat{\mathbf{R}} \mathbf{P}_{\mathbf{A}(\mathbf{z})}^{\perp}$, the ML height estimator is

$$
\hat{\mathbf{z}}_{N_{s}}=\arg \min _{\mathbf{z}_{\mathbf{N}_{\mathbf{s}}}} \alpha\left(\mathbf{z}_{N_{s}}\right)
$$

with

$$
\alpha\left(\mathbf{z}_{N_{s}}\right)=\left(\prod_{i=1}^{N_{s}} l_{i}^{S}\left(\mathbf{z}_{N_{s}}\right)\right)\left(\frac{1}{p-N_{s}} \sum_{i=1}^{p-N_{s}} l_{i}^{N}\left(\mathbf{z}_{N_{s}}\right)\right)^{p-N_{s}} .
$$

An algorithm based on alternating projections [8] is employed to solve this nonlinear, multimodal $N_{s}$-dimensional minimization problem.

\section{Polarimetric Multibaseline InSAR Spectral ANALYSIS ALGORITHMS}

In this section, the spectral analysis techniques are extended to the fully polarimetric MB InSAR configuration. In this situation, the antennas not only receive the signals in diverse polarizations [10], [7], but emit the electromagnetic waves and receive the echo in polarimetric mode. The following adaptation to the fully polarimetric case not merely increases the number of observables, but especially finds the optimal polarization combination for height estimation. Furthermore, these algorithms allow examining the scatterer physical properties by analysis of their polarimetric behavior.

\section{A. Polarimetric MB InSAR Signal Model}

The signal model for multibaseline InSAR data described in section II has to be generalized to take polarization diversity into account. The polarimetric multibaseline interferometric SAR received signal for $p$ sensors is modeled as:

$$
\begin{aligned}
\mathbf{y}(n) & =\sum_{i=1}^{N_{s_{t}}} \sqrt{\tau_{t_{i}}} \exp \left(j \psi_{i}\right) \mathbf{b}\left(z_{t_{i}}, \mathbf{k}_{t_{i}}\right) \\
& +\sum_{j=1}^{N_{s_{c}}} \sqrt{\tau_{c_{j}}} \mathbf{x}_{j}(n) \odot \mathbf{b}\left(z_{c_{j}}, \mathbf{k}_{c_{j}}\right)+\mathbf{v}(n) .
\end{aligned}
$$

The observation vector $\mathbf{y}(n) \in \mathbb{C}^{\tilde{p}}, \tilde{p}=4 p$, is a Gaussian random process with nonzero mean and covariance matrix $\mathbf{R} \in \mathbb{C}^{\tilde{p} \times \tilde{p}}$, i.e., $\mathbf{y}(n) \sim \mathcal{N}_{\mathbb{C}}(\boldsymbol{\mu}, \mathbf{R})$.

The main modification with respect to the single polarization model is the structure of the MB polarimetric interferometric (MBPI) steering vector $\mathbf{b}(z, \mathbf{k}) \in \mathbb{C}^{\tilde{p}}$. It is a linear combination of several steering vectors $\mathbf{a}_{\gamma_{i}}(z) \in \mathbb{C}^{\tilde{p}}$, each of them associated to one particular polarization:

$$
\mathbf{b}(z, \mathbf{k})=k_{1} \mathbf{a}_{\gamma_{1}}(z)+k_{2} \mathbf{a}_{\gamma_{2}}(z)+k_{3} \mathbf{a}_{\gamma_{3}}(z)+k_{4} \mathbf{a}_{\gamma_{4}}(z) .
$$

The weighting coefficients $k_{i} \in \mathbb{C}$ form a vector

$$
\mathbf{k}=\left[k_{1}, k_{2}, k_{3}, k_{4}\right]^{T} \in \mathbb{C}^{4}
$$

that may be interpreted as a scattering mechanism. This can be written in matrix notation as

$$
\mathbf{b}(z, \mathbf{k})=\mathbf{B}(z) \mathbf{k}
$$

with the matrix of MBPI steering vectors $\mathbf{B}(z) \in \mathbb{C}^{\tilde{p} \times 4}$

$$
\mathbf{B}(z)=\left[\mathbf{a}_{\gamma_{1}}(z), \mathbf{a}_{\gamma_{2}}(z), \mathbf{a}_{\gamma_{3}}(z), \mathbf{a}_{\gamma_{4}}(z)\right] .
$$

\section{B. Polarimetric MUSIC Algorithm}

If $N_{s}$ is the assumed number of scatterers, the matrix of the noise eigenvectors is $\mathbf{E}_{q} \in \mathbb{C}^{\tilde{p} \times q}$ with $q=\tilde{p}-N_{s}$. The pseudospectrum of MUSIC for the fully polarimetric SAR configuration is

$$
\hat{P}_{M U}^{F P}(z)=\frac{1}{\lambda_{\min }\left(\mathbf{B}^{H}(z) \mathbf{E}_{q} \mathbf{E}_{q}^{H} \mathbf{B}(z)\right)}
$$

with $\lambda_{\min }$ the smallest eigenvalue of the $4 \times 4$ Hermitian linear system

$$
\mathbf{B}^{H} \mathbf{E}_{q} \mathbf{E}_{q}^{H} \mathbf{B k}_{\min }=\lambda_{\min } \mathbf{k}_{\min } .
$$

The eigenvector $\mathbf{k}_{\min }$ describes the physical features of the scatterer and permits a polarimetric analysis [11]. The linear system (16) must be of full rank, otherwise $\lambda_{\min }=0$. This leads to an infinite pseudospectrum (15) and the height cannot be determined. A necessary criterion for the linear system having full rank is $\tilde{p} \geq N_{s}+4$. 


\section{Polarimetric MLE}

Let $\mathbf{C}(\mathbf{z})=\left[\mathbf{B}\left(z_{1}\right), \ldots, \mathbf{B}\left(z_{N_{s}}\right)\right]$ be the matrix of steering matrices (14) and the block diagonal polarization matrix $\mathbf{K} \in$ $\mathbb{C}^{4 N_{s} \times N_{s}}$

$$
\mathbf{K}=\operatorname{diag}\left[\mathbf{k}_{1}, \ldots, \mathbf{k}_{N_{s}}\right] .
$$

Using the abbreviation $\mathbf{D}=\mathbf{C}(\mathbf{z}) \mathbf{K}$, and keeping in mind that the matrix $\mathbf{D}$ depends both on height $\mathbf{z}$ and polarization $\mathbf{K}$, the projection matrix $\mathbf{P}_{\mathbf{D}} \in \mathbb{C}^{\tilde{p} \times \tilde{p}}$ onto the signal subspace is

$$
\mathbf{P}_{\mathbf{D}}=\mathbf{D}\left(\mathbf{D}^{H} \mathbf{D}\right)^{-1} \mathbf{D}^{H}
$$

and the orthogonal projection $\mathbf{P}_{\overline{\mathrm{D}}}^{\perp} \in \mathbb{C}^{\tilde{p} \times \tilde{p}}$ onto the noise subspace is

$$
\mathbf{P}_{\mathrm{D}}^{\perp}=\mathbf{I}-\mathbf{P}_{\mathbf{D}} .
$$

Utilizing the decompositions of the matrices $\mathbf{P}_{\mathbf{D}} \hat{\mathbf{R}} \mathbf{P}_{\mathbf{D}}$ and $\mathbf{P}_{\mathbf{D}}^{\perp} \hat{\mathbf{R}} \mathbf{P}_{\mathbf{D}}^{\perp}$, the ML height estimator is

$$
\left(\hat{\mathbf{z}}_{N_{s}}, \hat{\mathbf{K}}\right)=\arg \min _{\left(\mathbf{z}_{N_{s}}, \mathbf{K}\right)} \beta\left(\mathbf{z}_{N_{s}}, \mathbf{K}\right)
$$

with

$$
\beta\left(\mathbf{z}_{N_{s}}, \mathbf{K}\right)=\delta\left(\mathbf{z}_{N_{s}}, \mathbf{K}\right) \varepsilon\left(\mathbf{z}_{N_{s}}, \mathbf{K}\right)
$$

where

$$
\delta\left(\mathbf{z}_{N_{s}}, \mathbf{K}\right)=\prod_{i=1}^{N_{s}} l_{i}^{S}\left(\mathbf{z}_{N_{s}}, \mathbf{K}\right)
$$

and

$$
\varepsilon\left(\mathbf{z}_{N_{s}}, \mathbf{K}\right)=\left(\frac{1}{\tilde{p}-N_{s}} \sum_{i=1}^{\tilde{p}-N_{s}} l_{i}^{N}\left(\mathbf{z}_{N_{s}}, \mathbf{K}\right)\right)^{\tilde{p}-N_{s}} .
$$

\section{EXPERIMENTAL RESUlts}

To demonstrate the performance of the above introduced algorithms, the building layover is investigated using fully polarimetric dual-baseline InSAR observations. The baselines of approximately $10 \mathrm{~m}$ and $40 \mathrm{~m}$ lead to a height of ambiguity of $H_{a m b}^{s b} \approx 67.5 \mathrm{~m}$ and $H_{a m b}^{l b} \approx 15 \mathrm{~m}$, respectively. In the following, the pseudospectra were calculated in the range $\left[-30 \mathrm{~m}, \frac{3}{4} H_{a m b}^{s b} \mathrm{~m}\right]$. The pseudospectrum of the ML estimator is defined as $\hat{P}^{M L}=\frac{1}{\beta}$ with $\beta$ given by (21).

Figure 1 and Figure 2 depict the pseudospectra of a sample associated with wall-ground interaction computed by the single polarization (SP) and fully polarimetric (FP) MUSIC algorithm and ML estimator, respectively. A peak at about 0 $\mathrm{m}$ related to the ground is clearly detected. The sidelobes are suppressed to around $-7 \mathrm{~dB}$ or less by the MUSIC method with and without polarization diversity. The secondary maxima are reduced to less than $-40 \mathrm{~dB}$ and $-7 \mathrm{~dB}$ by the polarimetric and single polarization MLE, respectively.

Finally, a pixel at the end of the building layover is selected, i.e., a sample inside the building layover that is nearest to the sensor position. The pseudospectra of the SP and FP MUSIC algorithm and the FP MLE shown in Figure 3 and Figure 4 consist of two components: The first at about $18 \mathrm{~m}$ corresponding to the building roof and the second at around $0 \mathrm{~m}$ related to ground. The sidelobes are lowered to $-8 \mathrm{~dB}$ and $-35 \mathrm{~dB}$ or less, respectively. The SP ML pseudospectrum reveals a maximum at around $18 \mathrm{~m}$ and secondary peaks at $-3 \mathrm{~dB}$.

Summarizing, two sources inside one azimuth-range resolution cell of building layover are separated and their heights are estimated by the single polarization and fully polarimetric MUSIC technique and the polarimetric MLE.

\section{CONClusion}

This paper has introduced two array signal processing techniques to analyze fully polarimetric multibaseline interferometric SAR measurements. They optimize the polarizations for scatterer height estimation and permit the determination of their physical behavior. Using dual-baseline POL-InSAR observations, the proposed techniques solve the layover problem by separating two contributions within one azimuth-range resolution cell: The first component related to the ground, the second to the building roof.

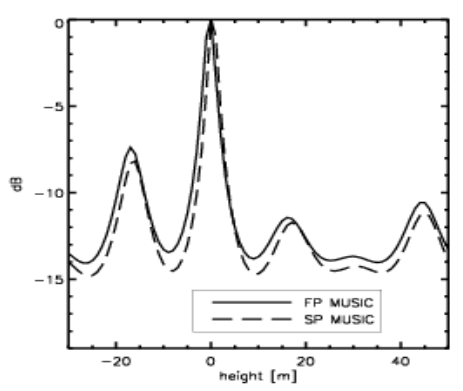

Fig. 1. Wall-ground interaction point: Single polarization and fully polarimetric MUSIC pseudospectra.

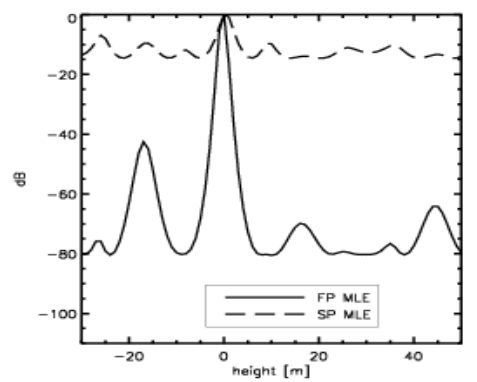

Fig. 2. Wall-ground interaction point: Single polarization and fully polarimetric MLE pseudospectra.

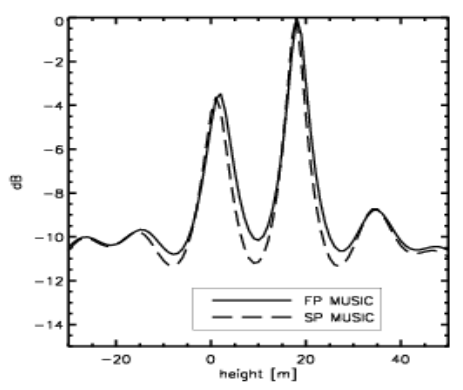

Fig. 3. Building layover sample: Single polarization and fully polarimetric MUSIC pseudospectra. 


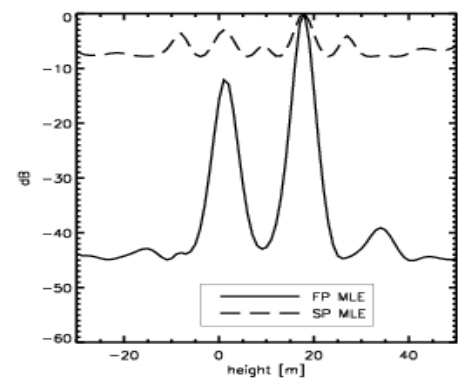

Fig. 4. Building layover sample: Single polarization and fully polarimetric MLE pseudospectra.

\section{REFERENCES}

[1] S. R. Cloude, K. P. Papathanassiou: Polarimetric SAR interferometry, IEEE Trans. Geoscience Remote Sensing, vol. 36, pp. 1551-1565, Sept. 1998.

[2] S. Guillaso, L. Ferro-Famil, A. Reigber, E. Pottier: Building Characterisation Using L-Band Polarimetric Interferometric SAR Data, IEEE Geoscience and Remote Sensing Letters, vol. 2, no.3, pp. 347-351, July 2005.
[3] S. Sauer, L. Ferro-Famil, A. Reigber, E. Pottier: $3 D$ Visualisation and Physical Feature Extraction of Urban Areas using Multibaseline POLInSAR Data at L-Band, Proceedings of URBAN'07, April 2007.

[4] S. Sauer, L. Ferro-Famil, A. Reigber, E. Pottier: Multibaseline POLInSAR Analysis of Urban Scenes for 3D Modeling and Physical Feature Retrieval at L-Band, Proceedings of IGARSS'07, July 2007.

[5] L. Ferro-Famil, A. Reigber, E. Pottier: Study and Applications of POLSAR Data Time-Frequency Correlation Properties, Proceedings of POLInSAR'07, Jan. 2007.

[6] F. Gini, F. Lombardini, M. Montanari: Layover Solution in Multibaseline SAR Interferometry, IEEE Trans. Aerospace Electronic Systems, vol. 38 , no. 4, pp. 1344-1356, Oct. 2002.

[7] R. O. Schmidt: Multiple Emitter Location and Signal Parameter Estimation, Proc. RADC Estimation Workshop, Rome Air Development Center, N.Y., Oct. 1979.

[8] M. Wax: Detection and Localization of Multiple Sources Via the Stochastic Signals Model, IEEE Trans. on Signal Processing, vol. 39, pp. 24502456, Nov. 1991.

[9] P. Stoica, A. Nehorai: MUSIC, Maximum Likelihood, and Cramer-Rao Bound, IEEE Trans. Acoustics, Speech, and Signal Processing, vol 37, pp.720-741, May 1989.

[10] E. R. Ferrara, T. M. Parks: Direction Finding with an Array of Antennas Having Diverse Polarizations, IEEE Trans. on Antennas and Propagation, vol. 31, pp. 231-236, March 1983.

[11] S. R. Cloude, E. Pottier: An Entropy Based Classification Scheme for Land Applications of Polarimetric SAR, IEEE Trans. Geoscience Remote Sensing, vol. 35, pp. 68-78, Jan. 1997. 